\title{
CLOROFILA a DO FITOPLÂNCTON EM SEIS ENSEADAS UTILIZADAS PARA O CULTIVO DE MOLUSCOS BIVALVES NO LITORAL DE SANTA CATARINA
}

\author{
L.A.O. PROENÇA \\ CTTMar - UNIVALI \\ Rua Uruguai, 458, Cx. Postal 360, CPE 88 302-202, Itajaí, Brasil. \\ e-mail: proenca@cttmar.univali.br
}

\begin{abstract}
RESUMO
A biomassa do fitoplâncton em seis enseadas do litoral catarinense utilizadas para o cultivo de moluscos marinhos foi avaliada por meio da clorofila a com a utilização da técnica de cromatografia líquida de alta eficiência (CLAE) ao longo de um ano. Os valores médios de Cla a foram relativamente baixos, variando entre 1,63 e 3,36 $\mu \mathrm{g} . \mathrm{I}^{-1}$, com o máximo de 10,57 $\mu \mathrm{g} . \mathrm{I}^{-1}$, observado na Praia Alegre, no município de Penha. O resultados indicam um grau geral de trofia moderado e similar para as enseadas estudadas.
\end{abstract}

Palavras chave: clorofila a, CLAE, cultivo de moluscos, biomassa, fitoplâncton.

\section{PHYTOPLANKTON CHLOROPHYLL a ALONG SIX BIGHTS USED FOR MUSSEL CULTURE IN THE LITTORAL OF SANTA CATARINA.}

\begin{abstract}
Phytoplankton biomass, as chlorophyll a was quantified along 6 bights used for mussel culture in the coastal area of Santa Catarina State. Chlorophyll $a$, determined by high performance liquid chromatography (HPLC), showed similar mean values among the sites, ranging from 1,63 to $3,36 \mu \mathrm{g} . \mathrm{l}^{-1}$, with a maximum for $10.57 \mu \mathrm{g} . \mathrm{l}^{-1}$ found in Praia Alegre, Penha, SC. The results indicates that all bight show a similar biomass and a moderate trophic grade.
\end{abstract}

Key words: chlorophyll a, HPLC, mussel culture, biomass, phytoplankton.

\section{INTRODUÇÃO}

O cultivo de moluscos marinhos vem crescendo em várias regiões do mundo. Uma das características desta atividade é a rapidez do desenvolvimento dos organismos aquáticos e baixos custos do investimento. O alimento, no caso de moluscos, é oriundo do próprio ambiente, sem nenhum custo adicional além da manutenção da estrutura de cultivo (Marenzi, 1999; Valenti et al. 2000). Em Santa Catarina a maricultura de moluscos bivalves, principalmente o mitilideo Perna perna, está definitivamente estabelecida. O estado já é o principal produtor do Brasil, com uma produção estimada em $12 \times 10^{3}$ ton. ano-1 (Valenti et al. 2000) Muito da expansão na produção é decorrente do sucesso de políticas direcionadas exercidas por universidades e órgãos públicos de fomento. O estímulo a atividade foi oriundo por um lado das quedas no rendimento da atividade pesqueira artesanal e de outro da necessidade de expansão na produção de alimentos. As- 
sim, diversos ambientes do litoral de Santa Catarina, entre estuários, baías e enseadas tem sido utilizados para o estabelecimento do cultivo de moluscos. É possível encontrar cultivos em enseadas próximas à foz de rios, como na foz dos rios Tijucas e Itajaí; dentro de baías ou estuários como, nas Baías Norte e Sul (Florianópolis) e Babitonga (São Francisco); ou áreas mais expostas ao oceano, como o caso da praia da Pinheira, ao sul de Florianópolis. Além de $P$. perna, também se cultivam ostras (Crassostera gigas e C. rhiizophorae) e vieira (Nodipectem nodosus), que esta sendo introduzida na região. Embora existam alguns cultivos fixos, o sistema mais utilizado é o de long-line, no qual os moluscos são cultivados suspensos por cabos conectados a bóias que oscilam com as variações das marés.

O cultivo de moluscos vem sendo alvo de constante discussão quanto aspectos econômicos, sociais e ambientais, fundamentais para a sustentabilidade de uma atividade relativamente nova no país (Vinatea, 1999; Valenti et al. 2000). A sustentabilidade depende da aplicação do conhecimento do funcionamento ecológico dos ambientes marinhos onde são efetuados os cultivos. Este conhecimento é fundamental para se avaliar os potenciais de produção e viabilidade. A capacidade suporte, ou seja, a quantidade de biomassa que pode ser mantida em um ecossistema varia conforme este esteja ou não em equilíbrio (Odum, 1983). Vários fatores estão envolvidos na avaliação desta propriedade, incluindo a produção total e a autodepuração. Variáveis ambientais que descrevem a produção total de uma enseada destinada ao cultivo incluem a morfologia da região, a produtividade primária, a hidrodinâmica local, taxas de troca com ambientes limítrofes ou interfaces e outros (Grant et al., 1995; Schettini et al.,1997; Proença \& Schettini, 1998 e Pereira Fo. et al.. 1998).

A disponibilidade de alimento, seja quantidade ou qualidade, ao longo do ano, está diretamente relacionada ao sucesso na produção de moluscos bivalves. Os moluscos retiram seu alimento da água em um processo de filtração da água com baixa seleção de partículas, havendo no entanto, diferenciação entre as frações orgânica e inorgânica (C.R. Resgalla $\mathrm{Jr}$, CTTMar/UNIVALI com. pessoal). O suprimento de partículas nutritivas em suspensão, fitoplâncton ou detritos, deve ser tal que assegure o crescimento dos indivíduos cultivados de forma satisfatória, segundo requisitos próprios da atividade. O fitoplâncton é o principal componente alimentar entre a fração orgânica das partículas em suspensão. Desta forma, sua biomassa, ou peso total dos organismos fotossintéticos do plâncton, expressa por unidade de volume ou área, reflete a disponibilidade instantânea de alimento na coluna de água. A biomassa do fitoplâncton assim definida não é uma variável facilmente determinada em amostras naturais. Assim, a determinação do pigmento fotossintético clorofila a ( $\mathrm{Cla} a$ ), tem sido utilizada amplamente na ecologia aquática como um dos mais importantes índices de biomassa do fitoplâncton. A popularidade deste índice como indicador da biomassa do fitoplâncton deriva do fato de esta ser exclusiva de organismos autotróficos fotossintéticos e ser sua estimativa relativamente fácil (Mantoura et al., 1997).

No presente trabalho a biomassa do fitoplâncton em 6 áreas de cultivo distribuídas em 3 importantes municípios catarinense na produção de moluscos no estado são comparadas e avaliadas. Os dados para o presente trabalho foram obtidos durante a realização do projeto multidisciplinar e multinstitucional $D e$ senvolvimento Sustentado da Produção de Moluscos, Atividade Sistema de Controle de Qualidade de Água e Produto, MMA/PNMA/ 96CV087 que visou contribuir ao ordenamento da atividade de maricultura no estado de Santa Catarina.

\section{MATERIAIS E MÉTODOS}

Foram coletadas amostras em 6 áreas de cultivo de moluscos em 3 municípios produtores do litoral centro norte de SC: Penha, 
Bombinhas e Governador Celso Ramos (Figura 1). Os locais de coleta foram selecionados em função da distribuição da produção em cada município e da disponibilidade operacional de coleta. Assim, foram coletadas as seguintes áreas: Penha, nas localidades de Praia Alegre e Armação do Itapocoroy; Bombinhas, nas localidades de Zimbros e Canto Grande; Governador Celso Ramos, nas localidades de Ganchos do Meio e Fazenda da Armação. As coletas foram realizadas em colaboração com Técnicos Extensionistas da EPAGRI, utilizando-se pequenas embarcações. As amostras foram tomadas a partir de 09/09/97 e se estenderam por um período de aproximadamente um ano com coletas aproximadamente quinzenais.

As coletas foram realizadas sempre no mesmo local, na parte mais externa do cultivo, em profundidades variáveis entre 4 e 6 metros. Em cada ponto de coleta foram retiradas amostras para determinação da biomassa do fitoplâncton e salinidade. In situ foram determi-

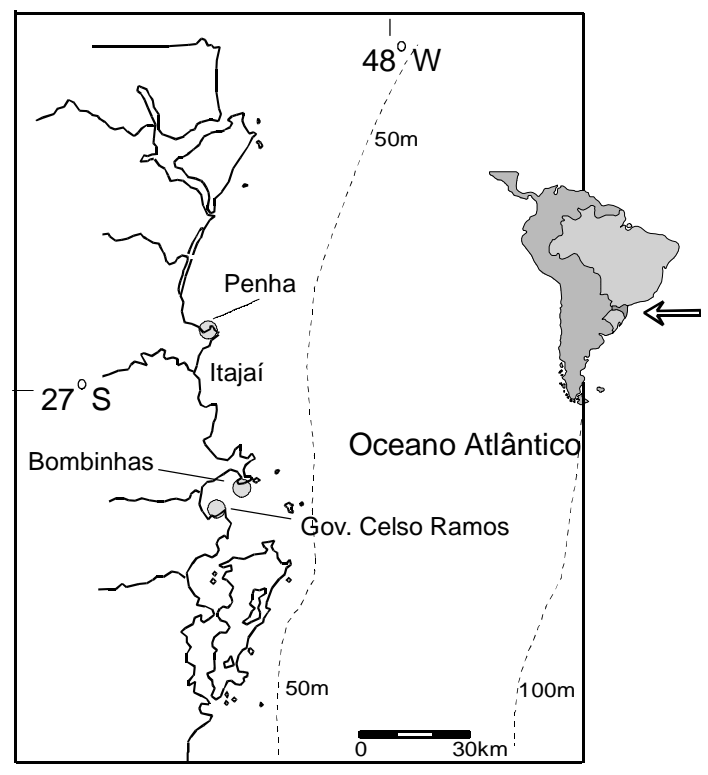

Figura 1: Área de estudos mostrando os municípios onde se desenvolveram as coletas. nadas a transparência e a temperatura da água, por meio de disco de Secchi e termômetro de mercúrio, respectivamente. A amostra para Cla a foi retirada utilizando-se um amostrador integral, confeccionado com uma mangueira de $1 / 2$ polegada com um registro em uma das extremidades e a outra aberta. A mangueira era baixada com o registro aberto até a profundidade desejada, por meio de um cabo fixo a um peso na extremidade inferior, de forma a permitir que a água da coluna penetrasse pela extremidade aberta. Assim que se atingia a profundidade desejada, o registro era fechado e a mangueira trazida a bordo. O material era então despejado em um balde, de onde se retiravam subamostras para determinação da Cla a e salinidade. Uma alíquota foi retirada para determinação da salinidade média da coluna de água por refratometria. $O$ valor da salinidade média foi posteriormente comparado com o valor da salinidade de superfície para verificação da presença de estratificação halina.

Para análise de Cla a uma alíquota de 50 a $100 \mathrm{ml}$ da amostra integral foi filtrada in situ em filtro de fibra de vidro de $25 \mathrm{~mm}$ de diâmetro (tipo Whatman GF/F) com um suporte de filtro acoplado a uma seringa de $50 \mathrm{ml}$. Os filtros foram dobrados, acondicionados em sacos plásticos e resfriados até o envio ao laboratório, no máximo até 12 horas após a coleta. Em laboratório, os filtros foram congelados até posterior análise por cromatografia líquida de alta eficiência (CLAE) conforme o método descrito a seguir.

A manipulação do filtro e do extrato foi feita em baixa intensidade luminosa. Ao filtro contendo a amostra foram adicionados $3 \mathrm{ml}$ de metanol grau HPLC. O filtro foi sonicado em sonda de ultrasom Sonics durante $40 \mathrm{~s}$. Durante a sonicação a temperatura foi mantida baixa por imersão do tubo em água gelada. Em seguida o extrato foi clarificado por filtração em filtro de fibra vidro. A cromatografia ocorreu em uma coluna cromatográfica Shimadzu SHIMPACK ${ }^{\circledR},(4,6 \mathrm{~mm}$ de diâmetro interno e $40 \mathrm{~mm}$ de comprimento) empacotada com partículas ODS de $3 \mu \mathrm{m}$ de diâmetro. A Cla a foi detec- 
tada por um fluorímetro Shimadzu RF551 ajustado para $435 \mathrm{~nm}$ de excitação e $660 \mathrm{~nm}$ para emissão. O registro foi feito em um integrador 6-R6A também da Shimadzu. A fase móvel consistiu de uma solução de 8:2 de metanol e acetona, de grau HPLC, que passou pela coluna em um fluxo de $0,7 \mathrm{ml} \cdot \mathrm{min}^{-1}$. Logo antes da análise, a $160 \mu \mathrm{l}$ de amostra foram adicionados $40 \mu \mathrm{l}$ de solução de acetato de amônia 1M. A solução foi misturada e injetada manualmente em um injetor Rheodine equipado com um loop de $100 \mu l$ (Mantoura et al. 1997).

A calibração foi feita injetando uma quantidade conhecida de Cla a pura (Sigma) padronizada por espectrofotômetria, conforme a equação tricromática, segundo o método descrito em Parsons et al. (1984). Para a padronização da solução de Cla $a$, o spectrofotômetro deve ter duplo feixe e resolução maior que $2 \mathrm{~nm}$. A concentração de Cla $a$ na solução padrão em $\mu \mathrm{g} \cdot \mathrm{ml}^{-1}$ (para cubeta de $1 \mathrm{~cm}$ ) foi dada por:

$C=11,85\left(E_{664}-E_{750}\right)-1,54\left(E_{647}-E_{750}\right)-0,08\left(E_{630}-E_{750}\right)$

onde $E_{i}$ é a absorbância nos comprimentos de onda i. A partir da solução padronizada, uma quantidade conhecida de Cla a em $\mu \mathrm{g}(\mathrm{m})$ foi injetada no cromatógrafo repetidas vezes para se obter o fator de resposta $f$ em função da área integrada $(A)$ :

$f=\mathrm{m} \cdot \mathrm{A}^{-1}$

A concentração de Cla a na amostra $\left(Q_{t}\right)$ foi calculada conforme a equação:

$\mathrm{Q}_{\mathrm{t}}\left(\mu \mathrm{g} . \mathrm{I}^{-1}\right)=f . \mathrm{A}_{p} . \mathrm{v}_{e} /\left(\mathrm{v}_{f} \mathrm{v}_{\mathrm{i}}\right)$,

onde $\mathrm{A}_{p}$ e área da Cla a na amostra, $\mathrm{v}_{e}$ o volume do extrato em $\mu \mathrm{l}, \mathrm{v}_{f}$ o volume filtrado da amostra em litros e $v_{i}$ volume da amostra injetada em $\mu \mathrm{l}(80 \mu \mathrm{l}$ se as condições descritas acima forem as mesmas).

\section{RESULTADOS E DISCUSSÃO}

De maneira geral, os ambientes amostrados são similares entre si em maior ou menor grau. São ambientes próximos a costa, rasos e abrigados em relação ao vento predominante e à ondulações mais intensas. Da mesma forma, todas as enseadas amostradas têm certa influência direta da descarga continental de água doce, pelo aporte de rios de tamanho variado ou pelo escoamento superficial. Assim, as variáveis analisadas refletiram esta condição de similaridade.

$\mathrm{O}$ aporte de água doce nas enseadas foi evidenciado pela salinidade, que variou entre 18 e 35 (Figura 2). A estação localizada na enseada de Praia Alegre, por exemplo, sofre ação direta da descarga do Rio Iriri, responsável pelas baixas salinidades observadas em superfície. Na Armação do Itapocoroy, além da presença de um pequeno córrego, existe a influência da pluma de descarga do rio Itajaí-açu, que sofre advecção em direção ao norte, influenciando a salinidade local (Proença \& Schettini, 1998 e Schettini et al., 1999). As localidades amostradas no Município de Bombinhas, Canto Grande e Zimbros e de Ganchos do Meio e no município de Gov. Celso Ramos, recebem influência da descarga do rio Tijucas. Já a Fazenda da Armação recebe pouca influência direta do aporte de água doce local, apresentando portanto um maior valor de salinidade.

Em diversas ocasiões a salinidade de superfície foi menor do que a salinidade integral, demonstrando que embora sejam as localidades áreas rasas, existe a possibilidade de estratificação halina. Este fato deve ser levado em consideração conforme os objetivos dos trabalhos a serem desenvolvidos nas localidades. A estratificação da coluna de água condiciona diversos processos, incluindo a produção primária. A estratificação também cria a possibilidade de que os mexilhões ao longo da 
corda de cultivo sejam expostos à diferentes condições considerando-se o eixo vertical. Neste caso, existe a possibilidade de acumulo diferenciado de ficotoxinas ou outros contaminantes conforme a posição do organismo na estrutura de cultivo. Como era de se esperar, as estratificações mais significativas ocorreram na região de cultivo de Praia Alegre, devido ao aporte direto do rio Iriri . A temperatura da água foi semelhante entre as localidades amostradas e apresentou um caráter sazonal de variação (Figura 3) já evidenciado em outros trabalhos na região costeira de Santa Catarina.
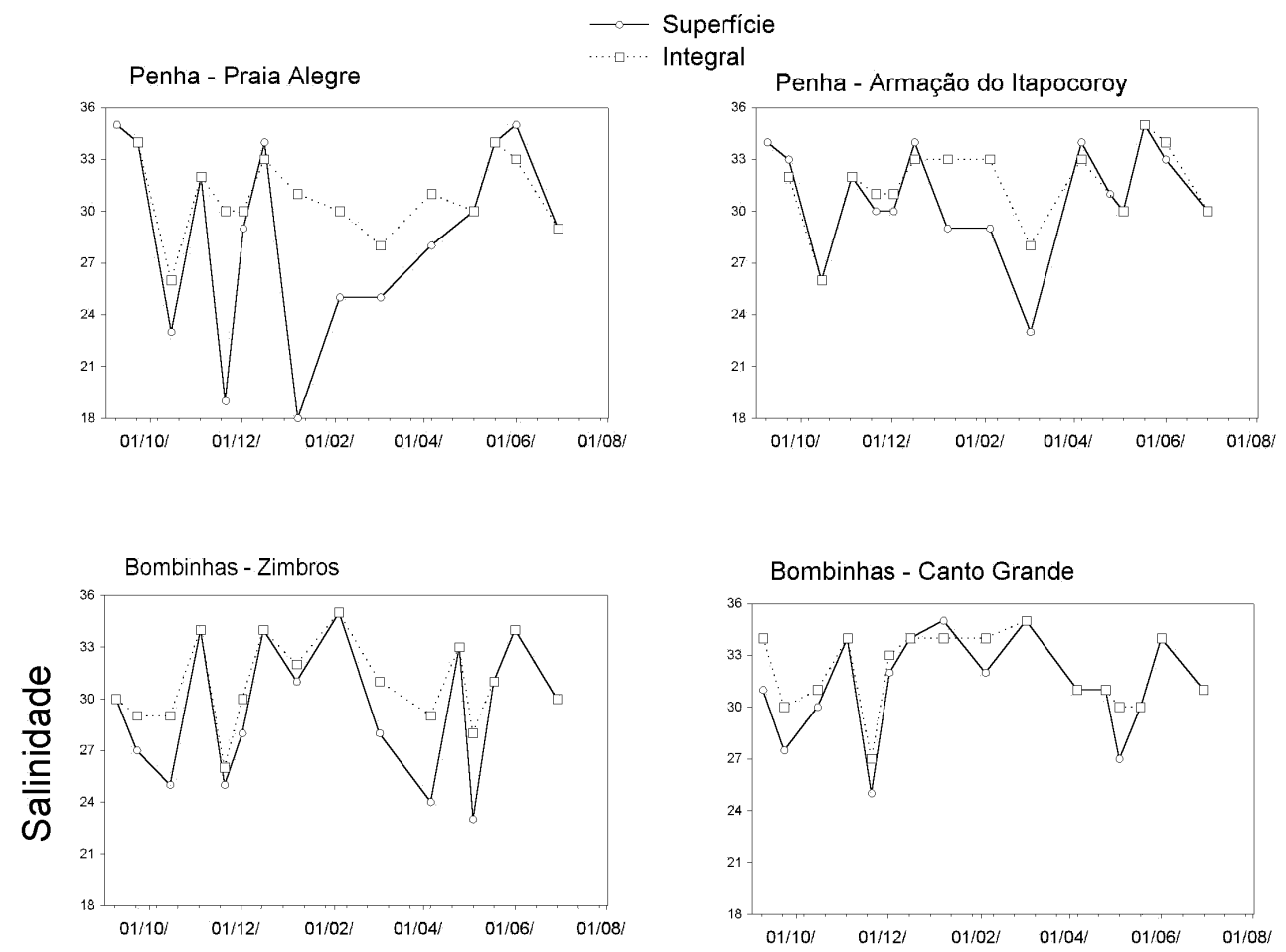

Gov. Celso Ramos - Ganho do Meio

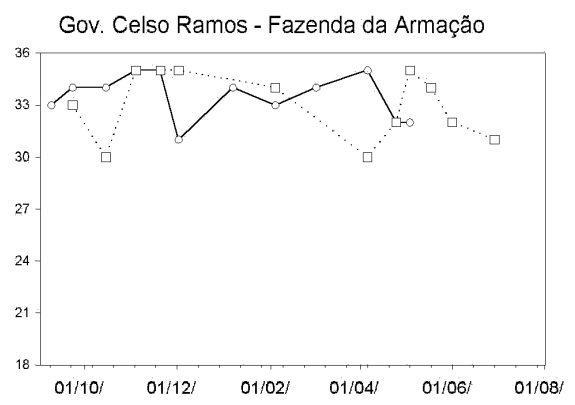

Figura 2: Salinidade da água de superfície e integral da coluna nas enseadas entre setembro de 1997e agosto de 1998. 
PROENÇA, L.A.O.: Clorofila a em enseadas utilizadas para o cultivo de moluscos.

A transparência da água variou entre as enseadas amostradas, sendo que os máximos e mínimos foram de 3,9 e 0,3 m, respectivamente (Figura 4). Os valores médios estiveram entre 2,6 e 1,2 m encontrados nas enseadas de Armação do Itapocoroy e Fazenda da Armação, respectivamente. Considerando-se que a profundidade de compensação, equivalente a zona eufótica (a profundidade onde a fotossíntese e a respiração da coluna de água são equivalentes), está na profundidade aproximadamente a 3 vezes a profundidade de disco de Secchi (Parsons et al., 1984), tem-se que em certas situações a produção primária

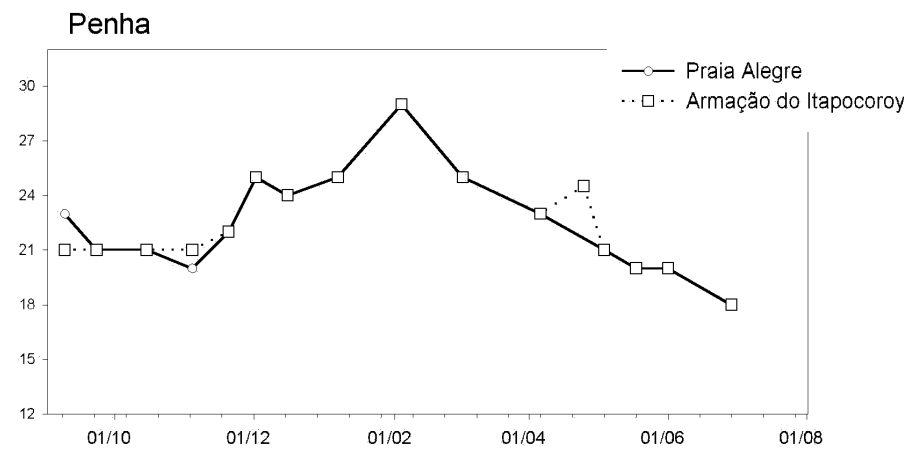

Bombinhas

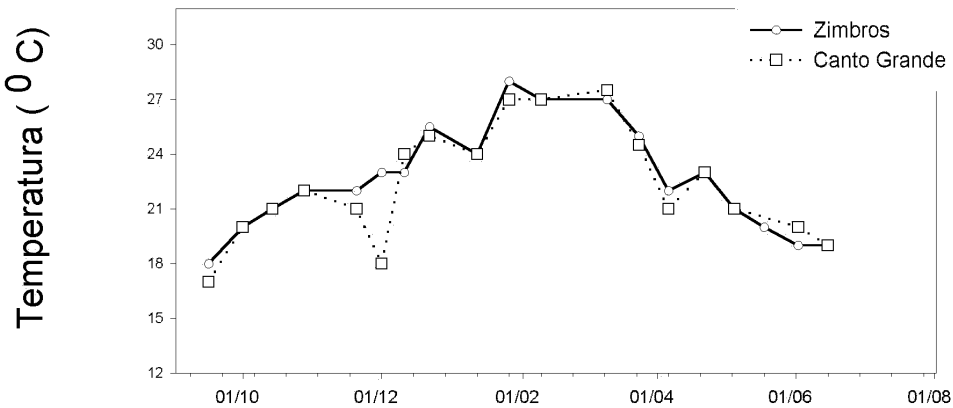

Governador Celso Ramos

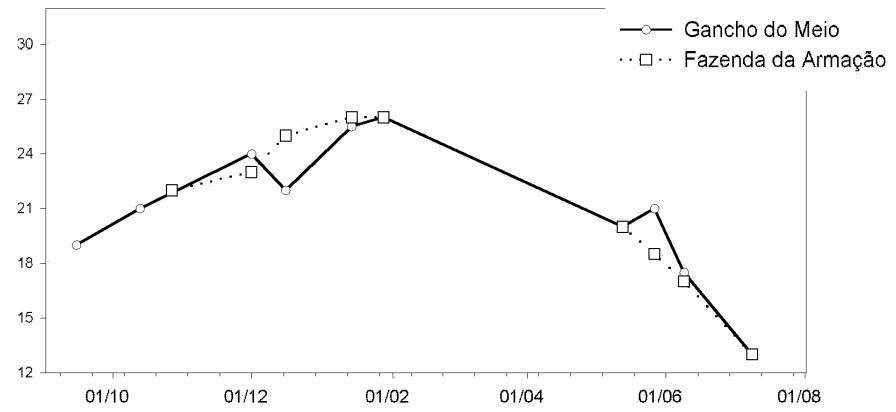

Figura 3: Temperatura da água da coluna nas enseadas entre setembro de 1997e agosto de 1998. 
do fitoplâncton nas enseadas pode ser limitada pela luz. Por exemplo, para uma coluna de água de $4 \mathrm{~m}$ de profundidade e disco de Secchi de $1 \mathrm{~m}$, a zona eufótica estaria restrita aos primeiros 3 metros de profundidade, indicando uma situação propensa a heterotrofia da coluna de água. A turbidez, que controla em parte a disponibilidade de luz na água, nas enseadas pode ser gerada pelo aporte continental, como no caso da pluma do rio Itajaí-açu, ou ressuspensão de material depositado nas enseadas (Proença \& Schettini, 1998; Schettini et al., 1997). Nestas circunstâncias, a estratificação da coluna de água se apresenta
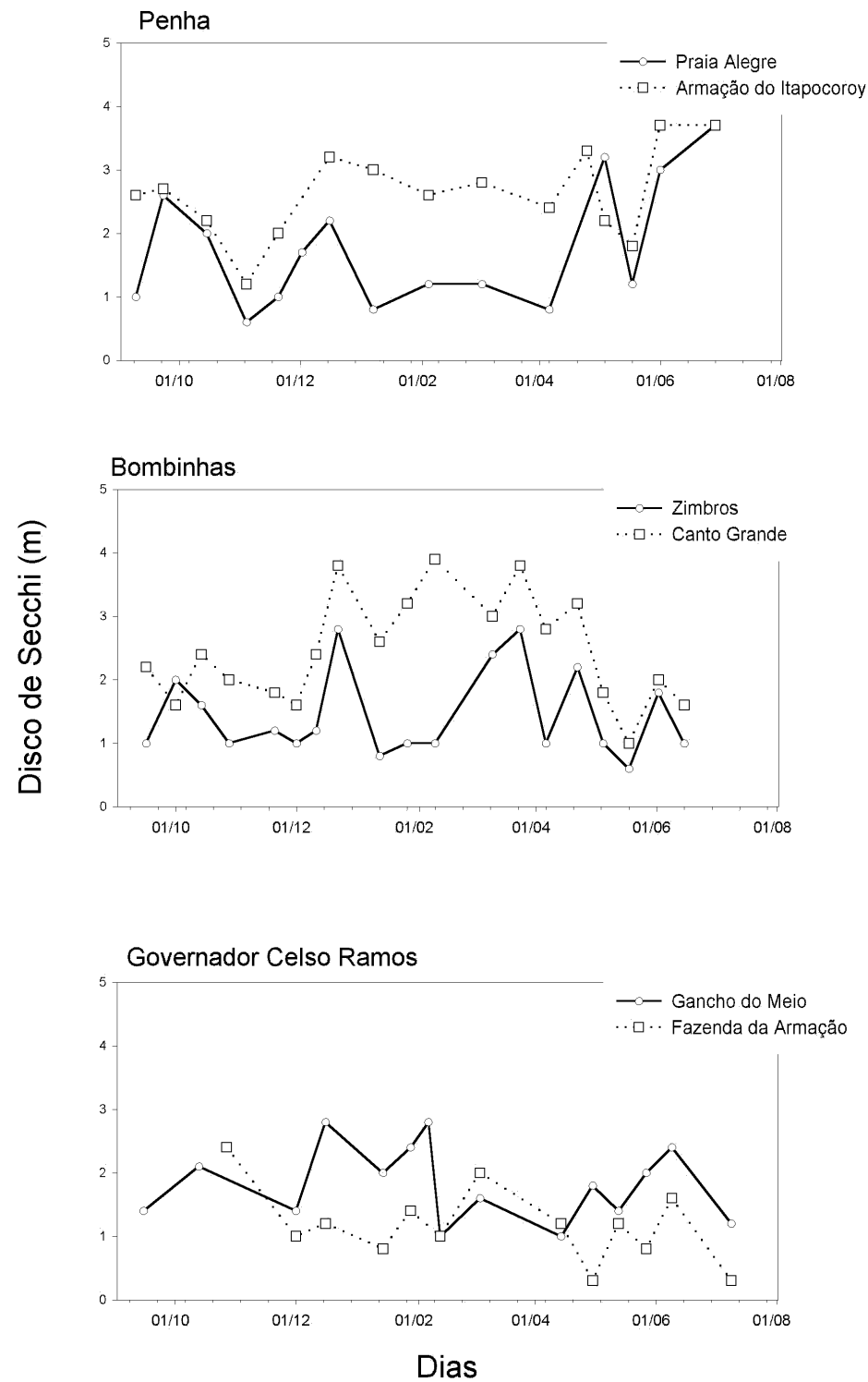

Figura 4:Transparência da água nas enseadas entre setembro de 1997e agosto de 1998. 
PROENÇA, L.A.O.: Clorofila a em enseadas utilizadas para o cultivo de moluscos.

como um mecanismo potencialmente importante na manutenção da biomassa local, por poder geral estabilidade da coluna de água.

A Figura 5 apresenta a variação da concentração de Cla a ao longo do tempo nas enseadas. O máximo e o mínimo registrados fo- ram de 10,53 e 0,19 $\mu \mathrm{g} . \mathrm{l}^{-1}$, na enseada de Praia Alegre, em 15/10/97 e enseada de Ganchos do Meio em 16/12/97, respectivamente. Os valores médios de Cla a foram em geral baixos e semelhantes (Tabela 1). Em um trabalho realizado em Armação do Itapocoroy, Chevarria

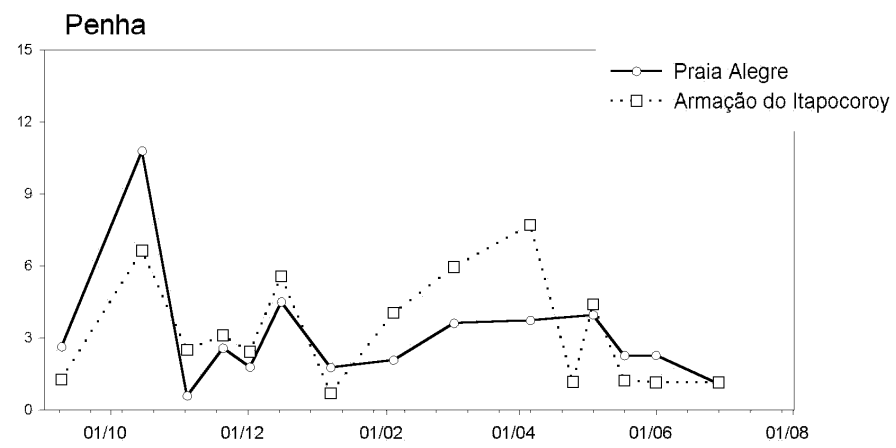

Bombinhas

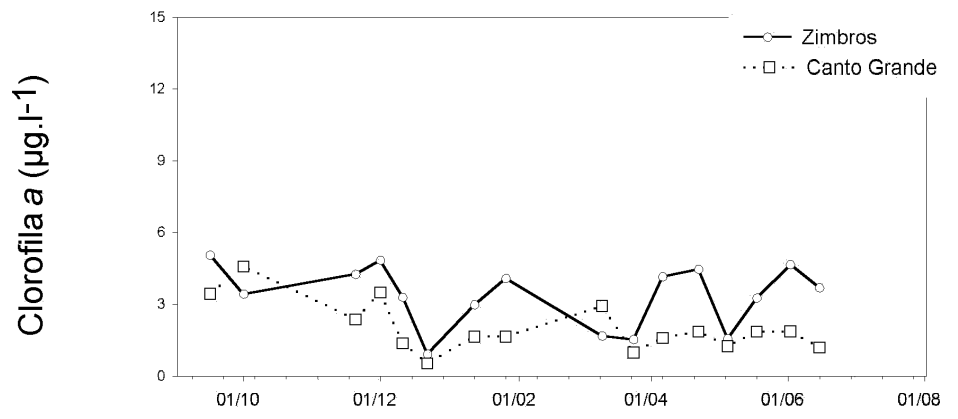

Governador Celso Ramos

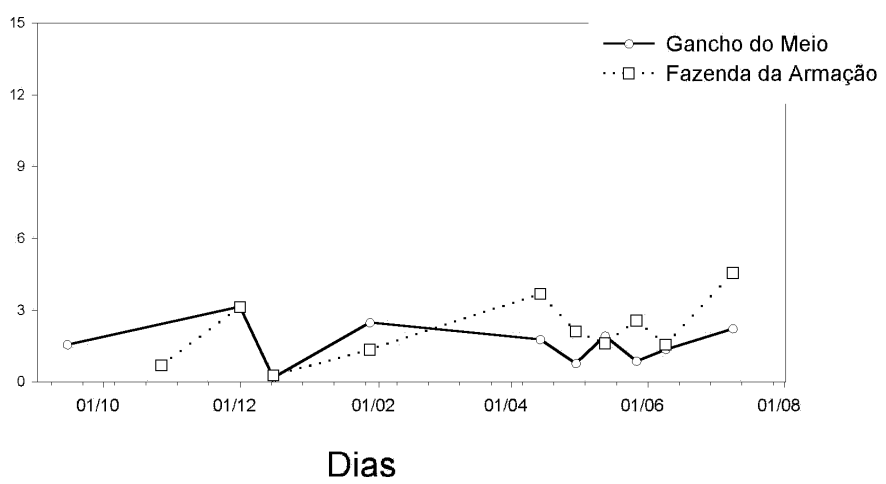

Figura 5: Concentração de clorofila a nas enseadas entre setembro de 1997e agosto de 1998. 
Tabela 1: Média (x), desvio padrão (s), número de amostras ( $\mathrm{n}$ ) e máxima concentração (max) das principais variáveis amostradas nas 6 enseadas de cultivo de moluscos estudadas nas enseadas entre setembro de 1997e agosto de 1998.
P. Alegre A Itapocoroy Zimbros
C. Grande
G. Meio F. Armação

\begin{tabular}{|c|c|c|c|c|c|c|c|}
\hline \multirow{3}{*}{$\begin{array}{l}\text { prof. Secchi } \\
\text { (m) }\end{array}$} & $x$ & 1,75 & 2,63 & 1,44 & 2,46 & 1,82 & 1,17 \\
\hline & $\mathrm{s}$ & 0,99 & 0,70 & 0,69 & 0,85 & 0,60 & 0,60 \\
\hline & $n$ & 15 & 15 & 19 & 19 & 15 & 13 \\
\hline \multirow{3}{*}{$\begin{array}{l}\text { salinidade } \\
\text { integral }\end{array}$} & $x$ & 28,67 & 30,81 & 29,74 & 31,18 & 31,67 & 32,82 \\
\hline & $S$ & 5,64 & 3,19 & 3,65 & 2,67 & 3,22 & 1,40 \\
\hline & $n$ & 15 & 16 & 19 & 19 & 15 & 11 \\
\hline \multirow{3}{*}{$\begin{array}{l}\text { salinidade } \\
\text { superfície }\end{array}$} & $x$ & 30,79 & 31,50 & 31,00 & 31,95 & 33,00 & 33,50 \\
\hline & $S$ & 2,29 & 2,41 & 2,33 & 2,12 & 1,95 & 1,31 \\
\hline & $n$ & 14 & 14 & 19 & 19 & 12 & 12 \\
\hline \multirow{3}{*}{$\begin{array}{l}\text { temperatura } \\
\text { da água }\end{array}$} & $x$ & 22,71 & 22,54 & 22,68 & 22,34 & 20,17 & 21,1 \\
\hline & s & 2,77 & 2,70 & 2,82 & 3,18 & 3,85 & 4,42 \\
\hline & $n$ & 17 & 17 & 20 & 20 & 17 & 14 \\
\hline \multirow{4}{*}{$\begin{array}{l}\text { clorofila a } \\
\mu \mathrm{g} . \mathrm{I}^{-1}\end{array}$} & $x$ & 3,10 & 3,25 & 3,36 & 2,03 & 1,63 & 2,15 \\
\hline & $\max$ & 10,57 & 7,69 & 5,05 & 4,57 & 3,14 & 4,55 \\
\hline & $s$ & 2,47 & 2,31 & 1,30 & 1,07 & 0,88 & 1,34 \\
\hline & $\mathrm{n}$ & 14 & 15 & 16 & 16 & 10 & 10 \\
\hline
\end{tabular}

\& Kuroshima (1998) encontraram um valor médio de 2,8 $\mu \mathrm{g} . \mathrm{I}^{-1}$ de Cla a durante aproximadamente 2,5 anos de amostragem. A ausência de um sinal claro de sazonalidade na biomassa também foi característica dos dados e esta de acordo com trabalhos anteriores na região. As concentrações de $\mathrm{Cla}$ a observadas nas 6 enseadas estão entre os valores reportados em diferentes ambientes costeiros do Brasil (Brandini et al., 2000 e trabalhos aí referenciados). Alguns estuários, no entanto, apresentam valores bastante elevados, como por exemplo entre 80 e $50 \mu \mathrm{g} . \mathrm{l}^{-1}$ de Cla $a$, observados na Lagoa dos Patos - RS e da Conceição - SC, respectivamente. Comparada com a região da plataforma costeira adjacente, onde os valores de Cla a são bastante baixos e variam entre 0,05 e 0,5 $\mu \mathrm{g} . \mathrm{I}^{-1}$ de Cla a (Brandini et al., 2000), podemos concluir que as enseadas estudadas, que apresentaram médias entre 1,6 e 3,3 $\mu \mathrm{g} . \mathrm{I}^{-1}$, possuem um grau de trofia moderado.

As enseadas estudadas aparentemente não são diferentes o suficiente para imprimir condições para o estabelecimento de diferentes biomassas do fitoplâncton (Tabela 1). A única exceção foi Ganchos do Meio, que apresentou biomassa média significativamente menor que as outras localidades $(p=0,047 \mathrm{em}$ teste não paramétrico de Kuskall-Wallis). Isto indica que, em geral, do ponto de vista da biomassa, estas enseadas respondem de maneira similar aos fatores condicionantes. Talvez esta condição seja reflexo dos padrões de circulação que dominam o litoral centro norte de Santa Catarina. Carvalho et al. (1998) descrevem dois padrões de distribuição de massa de água típicos para a área: situações de primavera/verão e outono/inverno. A primeira é característica pela coluna de água dividida em 
duas camadas, com termoclina bastante acentuada. A possibilidade de ressurgência da Água Central do Atlântico Sul (ACAS) por ação de ventos do quadrante $\mathrm{NE}$, pode trazer águas mais salinas, frias, ricas em nutrientes próximo à zonas de cultivo. Na segunda situação, a coluna de água é mais homogênea, em conseqüência da subsidência da água costeira por ação de ventos mais persistentes do quadrante sul e advecção de águas de origem subantártica, influenciada pelo aporte continental dos estuários do Rio da Prata e Lagoa dos Patos. A água costeira influencia as enseadas com baixa salinidade e elevado material em suspensão (Schettini et al., 1999).

O crescimento de moluscos nos cultivos esta diretamente relacionado à disponibilidade de alimento do ambiente que é função de dois fatores básicos: taxa de produção primária local (autóctone) e taxa de fornecimento de matéria orgânica oriunda de outras regiões (alóctone). A primeira, esta intimamente relacionada a disponibilidade local de nutrientes, como fósforo e nitrogênio e disponibilidade de radiação. Segundo trabalhos realizados na Armação do Itapocoroy (Chevarria \& Kuroshima, 1998; Pereira Fo. et al., 1998), aparentemente, não existe limitação generalizada do crescimento do fitoplâncton por falta de nutrientes. As análises feitas apontam concentrações relativamente altas de nitrogênio (nitrato e amônia), fosfato e silicatos. Por outro lado, pode haver limitação do fitoplâncton por luz, conforme observado no presente trabalho. A taxa de fornecimento alóctone de matéria orgânica, por sua vez, esta intimamente relacionada a processos advectivos. Trabalhos sobre correntes realizados na enseada de Armação do Itapocoroy apontam para uma alta taxa de renovação da água, indicando que a advecção é um processo importante na manutenção dos valores de biomassa observados (Schettini et al., 1999). A dependência do aporte alóctono para a manutenção da produção de moluscos na enseada de Armação do Itapocoroy foi apontada também em um trabalho onde se quantificou o fluxo vertical de fitodetritos sob os cultivos (Proença \& Schettini, 1998).

Observou-se uma tendência de uma relação inversa entre a salinidade e a Cla a. Esta tendência ficou evidente em uma análise de componentes principais com a entrada de 78 observações (Figura 6). A salinidade integral da coluna de água e a Cla a foram as principais variáveis e estiveram em lados opostos do Eixo2, que explica $26 \%$ da variação encontrada nos dados. A oposição destas duas variáveis no eixo, sugere que uma menor salinidade condicione uma maior biomassa. Uma possibilidade para explicar esta relação é que o aporte de água doce e pode favorecer a estabilidade da coluna de água, causando estratificação, o que aumentaria a exposição da comunidade do fitoplâncton à luz. Assim, é de se esperar que exista uma relação direta da biomassa com o aporte de água doce, condicionado pela chuva na bacia de drenagem local ou pela advecção de pluma de rios, como ocorre com o rio Itajaí-açu (Schettini et al., 1999).

A medida que o cultivo de molusco se intensifica em uma determinada região, a taxa

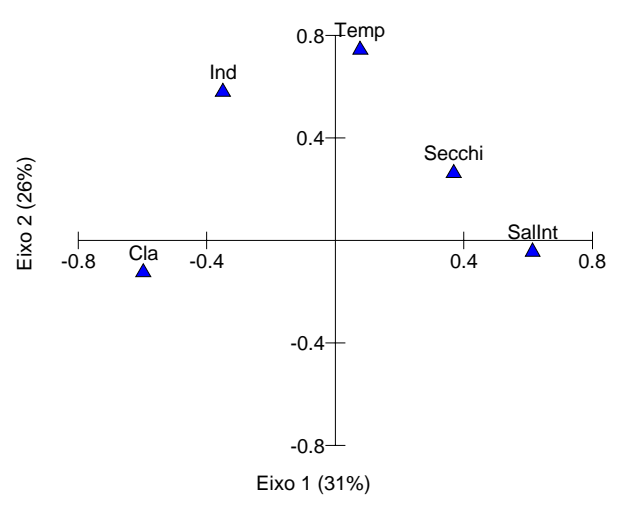

Figura 6: Resultado da análise de componentes principais, com entrada de 78 casos, mostrando os dois eixos principais, responsáveis por $55 \%$ da variabilidade dos dados. Clorofila a (Cla), temperatura (Temp), transparência da água (Secchi), salinidade (Sallnt) e índice de estratificação (Ind, diferença entre a salinidade integral e de superfície, para detalhes ver texto). 
total de filtração dos organismos, com conseqüente retirada de biomassa da água, torna-se um fator fundamental para a manutenção da biomassa disponível (Proença \& Schettini, 1998). Em certos casos, a taxa de retirada da biomassa é tanta que chega a limitar o crescimento dos moluscos por falta de alimento, conforme observado em localidades onde 0 cultivo é demasiadamente denso. Um reflexo direto é o aumento da taxa de sedimentação local associada a perda da qualidade de água (Grant et al., 1995) . Considerando que a produtividade primária do fitoplâncton nas enseadas seja controlada por fatores que atuam em uma escala maior, como padrões de circulação ou descarga dos rios, a taxa de fornecimento alóctone de biomassa, função da hidrografia, se torna uma variável chave para a manutenção da produtividade nos cultivos de moluscos.

\section{AGRADECIMENTOS}

A Nagib Paulo de Souza e Marcio Silva Tamanaha pelo auxílio nas análises no laboratório e a todo o pessoal técnico da Epagri, que atua junto às comunidades, pela coleta e envio das amostras. A Katia Naomi Kuroshima e Charrid Resgalla Jr. pela revisão do manuscrito. Este trabalho foi financiado pelo projeto multidisciplinar e multinstitucional Desenvolvimento Sustentado da Produção de Moluscos, Atividade Sistema de Controle de Qualidade de Água e Produto, convênio MMA/PNMA/ 96CV087.

\section{REFERÊNCIAS}

Brandini, F.P.; Silva A.S. \& L.A.O. Proença, 2000. Oceanografia e maricultura. In: Valenti, W.C.; Poli, C.R.; Pereira, J.A. \& J.R. Borghetti (Eds), Aqüicultura no Brasil: bases para um desenvolvimento sustentável, MCT/CNPq, Brasília. p. 107-141.
Carvalho, J.L.B.; Schettini, C.A. \& T.M. Ribas. 1998. Estrutura termohalina do litoral centro-norte catarinense. Notas Téc. FACIMAR, 2: 181-19.

Chevarria, G.G \& K.N. Kuroshima. 1998. Monitoramento químico de uma área de cultivo de moluscos - Enseada de armação de Itapocoroy. Penha SC. XI Semana Nacional de Oceanografia, Rio Grande, RS, 18-24 de novembro, 544-546.

Grant, J.A.; Hatcher, A.; Scott, D.B. ; Pocklington, P.; Schafer C.T. \& G.V. Winters. 1995. A multidisciplinary approach to evaluating impacts of shellfish aquaculture on benthic communities. Estuaries, 18: 124-144.

Mantoura F.A., Jeffrey, S.W.; Llewellyn, C.A.; Claustre, H. \& C.E. Morales. 1997. Comparison between spectrophotometric, fluorometric and HPLC methods for chlorophyll analysis. In: Jeffrey, S.W.; Mantoura R.F.C. \& S.W. Wright (Eds.). Phytoplankton pigments in oceanography, UNESCO, Paris. p. 361-380.

Marenzi, A.W.C. 1999. O uso de mecanização nos cultivos de mexilhão Perna perna em Santa Catarina. Agrárias 18:155-163.

Odum, E.P. 1983. Basic Ecology. Saunders College Publishing, Philadelphia. 613p.

Parsons, T.R., Takahashi, M. \& B. Hargrave. 1984. Biological oceanographic processes. Pergamon Press, Oxford. 330p.

Pereira Fo., J.; Oliveira U.C. \& G.C. Manzoni. 1998. O uso de Bell jar na avaliação do metabolismo bentônico na Armação do Itapocoroy: resultados preliminares. Notas Téc. FACIMAR 2: 81-92.

Proença, L.A.O. \& C.A. Schettini. 1998. Effect of shellfish culture on phytodetritus vertical fluxes in tropical waters - Southern Brazil. Revista Brasileira de Oceanografia, 46:125133.

Schettini, C.A.F., Resgalla, C. Jr \& K.N. Kuroshima. 1997. Avaliação preliminar da taxa de sedimentação na região de cultivo de moluscos na Enseada da Armação, SC. Notas Téc. FACIMAR, 1: 1-7. 
PROENÇA, L.A.O.: Clorofila a em enseadas utilizadas para o cultivo de moluscos.

Schettini, C.A.F.; Carvalho, J.L.B \& E. Truccolo. 1999. Aspectos hidrodinâmicos da armação de Itapocoroy, SC. Notas Téc. FACIMAR, 3: 99-109.

Valenti, C.W.; Poli, C.R.; Pereira, J.A. \& J.R. Borghetti. 2000. Aqüicultura no Brasil: ba- ses para um desenvolvimento sustentável. MCT/CNPq, Brasília. 398p

Vinatea, L.A. 2000. Aqüicultura e desenvolvimento sustentável. Editora da UFSC. 310p. 